\title{
Identification and characterization of lipid droplets in Malassezia furfur
}

\author{
Celis AM1,2,3, Triana $\mathrm{S}^{2,3}$, Ibarra H, Cardona J4, Restrepo S², Gonzalez A4, Wösten HAB¹, de Cock H¹
}

${ }^{1}$ Microbiology, Department of Biology, Utrecht University, Utrecht, The Netherlands, 2Laboratorio de Micología y Fitopatología, Departamento de Ciencias Biológicas Universidad de Los Andes, Bogotá, Colombia, ${ }^{3}$ Grupo Investigación Celular y Molecular de Microorganismos Patógenos (CeMoP), ${ }^{4}$ Grupo de Diseño de Productos y Procesos (GDPP), Departamento de Ingeniería Química, Universidad de los Andes

Background: Malassezia furfur is a lipid-dependent yeast that is part of the human skin microbiota. Mechanisms underlying growth on skin including its lipid metabolism are largely unknown.

Methods: We followed physiological characterization of M. furfur in complex medium (mDixon) and minimal medium suplemmented with sources of palmitic acid and/ or oleic acid to evaluate the presence of the lipid droplets (LD) throught confocal microscopy using nile red staining. Lipidomic and genomic analysis were conducted in order to characterize the lipid composition and to identify the presence of the genes involved in neutral lipid synthesis in this yeast.

Results: Lipid droplets (LDs) are shown in Malassezia. Lipidomic analysis revealed the presence of phospholipids as well as TGs in LDs of $M$. furfur but SEs were not detected. TG species 52:4, 54:3, 54:4, and 54:5 were enriched in LDs after growth in the presence of Tween 80 or oleic acid (both donors of C18:1), while TG species 50:1 and 52:2 were enriched after growth in the presence of Tween 40 (donor of C16:0).

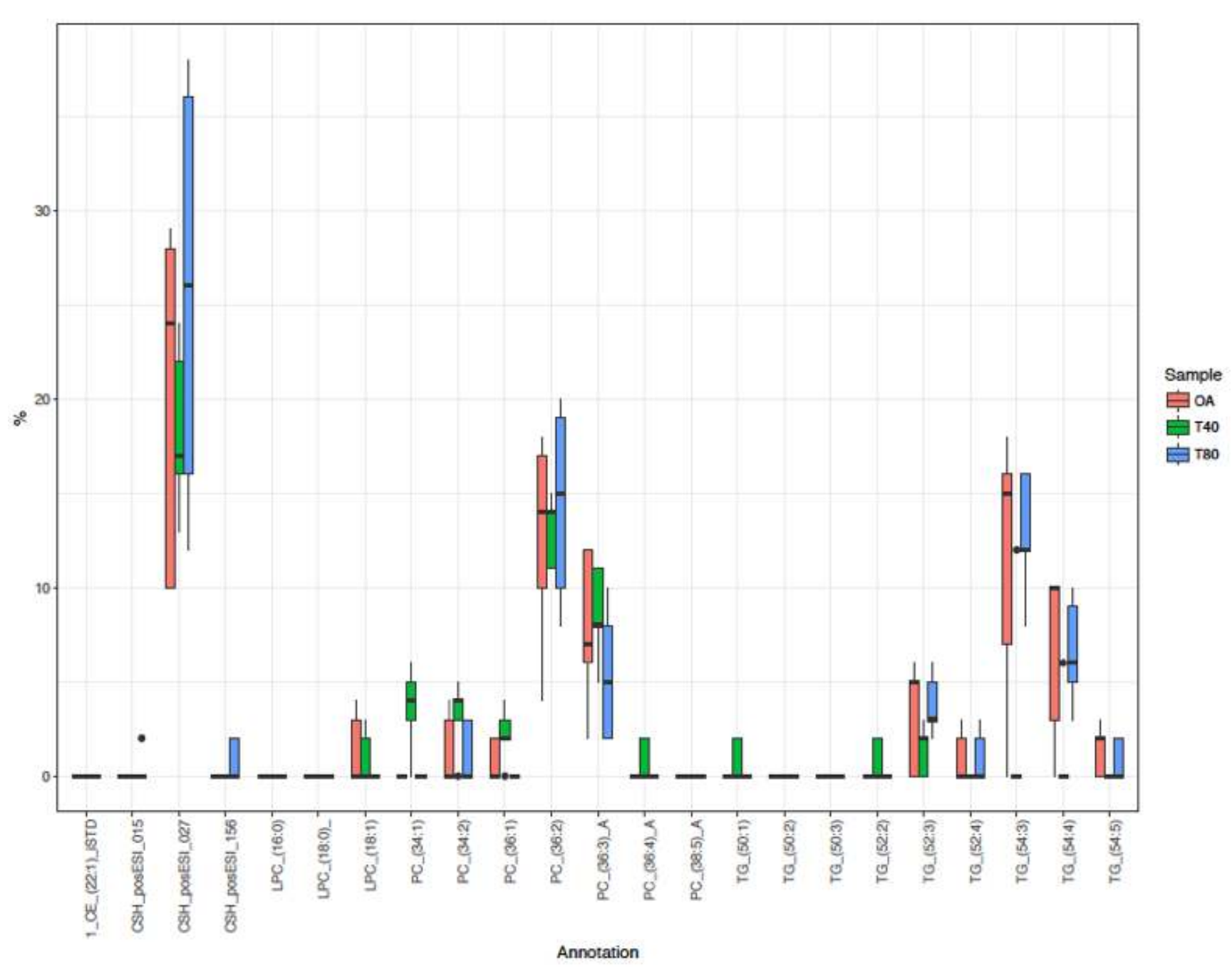

Figure 2. Triglycerides (TG), phosphatidylcholine (PC), and lysophosphatidylcholine (LPC) profiles of LDs isolated from $M$. furfur CBS1878 cultures grown in mDixon broth containing oleic acid (red bars) Tween 40 (green bars) or Tween 80 (blue bars). Error bars indicate standard deviation.

C.W. Wang, Lipid droplet dynamics in budding yeast, Cell. Mol. Life Sci., 72 (2015) 2677-2695.

Celis Ramirez, A. M., Wösten, H. A. B., Triana, S., Restrepo, S., \& de Cock, J. J. P. A. (2017). Malassezia spp. beyond The Mycobiota. SM Dermatology Journal, 3(3).
A

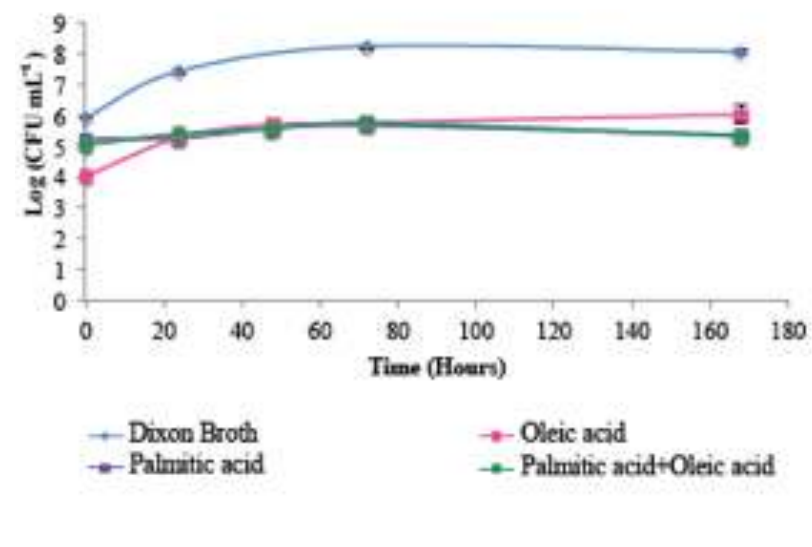

B
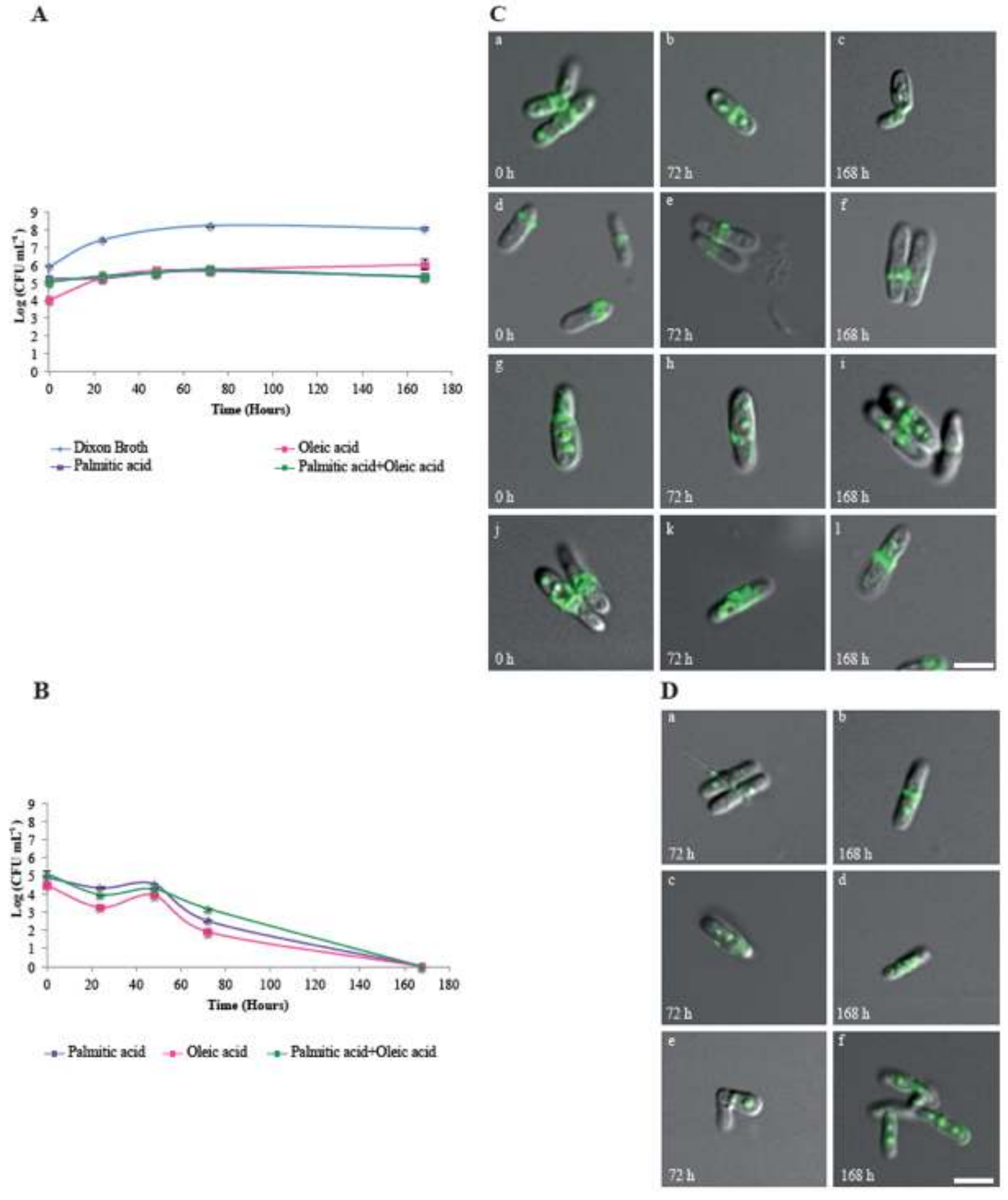

Figure 1. Growth curves (A, B) and localization of LDs (C, D) of $\quad M$. furfur CBS1878 in mDixon and / or MM with palmitic acid and / or oleic acid $(A, C)$ or after transfer to MM without fatty acids $(B, D)$. LDs were localized in cells by Nile Red staining 0,72 , and $168 \mathrm{~h}$ after culturing in mDixon (a-c) or in MM supplemented with PA (d-f) OA (g-i), or PA+OA (j-I) (C). Alternatively, LDs were localized in cells that had been grown for $168 \mathrm{~h}$ in $\mathrm{MM}$ with $\mathrm{PA}$ and / or OA and that were subsequently depleted for PA (a-b), OA (b-c) or PA+OA (d-e) (D). Bar represents 5 $\mu \mathrm{m}$.

Conclusions: Results suggest that LDs of $M$. furfur relieve lipotoxicity caused by oleic acid. This would compensate for the inability of this yeast to degrade unsaturated fatty acids via $\beta$-oxidation, which is likely explained by the absence of a gene encoding $\Delta 3,2$-enoyl-CoA isomerase.

General significance: We are presenting here new insights in the lipid metabolism of this yeast with specific attention to lipid droplets which is important to understand aspects in the adaptation to the host. 